Notre Dame Journal of Formal Logic

Volume 47, Number 3, 2006

\title{
Geometrical Axiomatization for Model Complete Theories of Differential Topological Fields
}

\author{
Nicolas Guzy and Cédric Rivière
}

\begin{abstract}
In this paper we give a differential lifting principle which provides a general method to geometrically axiomatize the model companion (if it exists) of some theories of differential topological fields. The topological fields we consider here are in fact topological systems in the sense of van den Dries, and the lifting principle we develop is a generalization of the geometric axiomatization of the theory $D C F_{0}$ given by Pierce and Pillay. Moreover, it provides a geometric alternative to the axiomatizations obtained by Tressl and Guzy/Point in separate papers where the authors also build general schemes of axioms for some model complete theories of differential fields. We first characterize the existentially closed models of a given theory of differential topological fields and then, under an additional hypothesis of largeness, we show how to modify this characterization to get a general scheme of first-order axioms for the model companion of any large theory of differential topological fields. We conclude with an application of this lifting principle proving that, in existentially closed models of a large theory of differential topological fields, the jet-spaces are dense in their ambient topological space.
\end{abstract}

\section{Basic Algebraic Geometry}

In what follows $K$ is a field of characteristic zero, $\Omega$ is a sufficiently saturated elementary extension of $K$ (in particular, $\Omega$ is of infinite transcendence degree over $K$ ), and $\widetilde{\Omega}$ denotes the algebraic closure of $\Omega$. We first recall some elementary definitions and results from algebraic geometry (for more details, see [9]). Let $J$ be an ideal of $K\left[X_{1}, \ldots, X_{n}\right]$ (also denoted $K[\bar{X}]$ when $n$ is understood); the sets $V(J):=\left\{\bar{a} \in \widetilde{\Omega}^{n} \mid \forall p \in J, p(\bar{a})=0\right\} \subseteq \widetilde{\Omega}^{n}$ are called $K$-Zariski-closed subsets. Moreover, a $K$-Zariski-closed set is $K$-irreducible if it is not the union of two disjoint proper $K$-Zariski-closed subsets. One can easily see that $V(J)$ is $K$-irreducible

Received February 9, 2006; accepted April 11, 2006; printed November 14, 2006 2000 Mathematics Subject Classification: Primary, 03C10; Secondary, 03C68

Keywords: model companion, topological fields, geometric axiomatization (C)2006 University of Notre Dame 
if and only if the ideal $I(V):=\left\{p \in K\left[X_{1}, \ldots, X_{n}\right] \mid p(\bar{a})=0\right.$ for all $\left.\bar{a} \in V(J)\right\}$ is prime. In this case $V(J)$ is called a $K$-variety.

Let $V$ be a $K$-variety; if $\bar{c} \in \widetilde{\Omega}^{n}$ is such that its $K$-Zariski-closure is equal to $V$, we say that $\bar{c}$ is a generic point for $V$. Equivalently, $\bar{c}$ is generic for $V$ if and only if the transcendence degree of $K(\bar{c})$ over $K$ is maximal among the points of $V$. In this case we define the dimension of $V, \operatorname{dim}(V)$, to be this transcendence degree.

We assume, furthermore, that $K$ is equipped with a derivation $D_{K}$. Let $\bar{a} \in V$; the torsor of $V$ at $\bar{a}$ is the set

$\tau_{\bar{a}}(V)=\left\{\bar{v}=\left(v_{1}, \ldots, v_{n}\right) \in \tilde{\Omega}^{n} \mid p^{D_{K}}(\bar{a})+\sum_{i=1}^{n} \frac{\partial p}{\partial X_{i}}(\bar{a}) \cdot v_{i}=0\right.$ for all $\left.p \in I(V)\right\}$,

where $p^{D_{K}}$ is the polynomial obtained by taking the derivative of the coefficients of $p$. We also define the torsor bundle of $V$ to be

$$
\tau(V)=\left\{(\bar{a}, \bar{v}) \in \tilde{\Omega}^{2 n} \mid \bar{a} \in V \wedge \bar{v} \in \tau_{\bar{a}}(V)\right\} .
$$

Let us remark that in the equations defining $\tau_{\bar{a}}(V)$ and $\tau(V)$ we may replace the condition "for all $p \in I(V)$ " by the condition "for all $p \in G$ " where $G$ is any set of generators of $I(V)$. Since $K[\bar{X}]$ is noetherian it follows that $\tau_{\bar{a}}(V)$ and $\tau(V)$ are $K$-definable (i.e., definable with parameters from $K$ ) in the language $\mathcal{L}_{\text {fields }}:=\left\{+,-, \cdot,^{-1}, 0,1\right\}$.

We end this section with the following important result due to Pierce and Pillay.

Lemma 1.1 Let $\bar{a} \in \widetilde{\Omega}^{n}$ be a generic point of a $K$-variety $V, \bar{b} \in \tau_{\bar{a}}(V)$, and $W=V(I(\bar{a}, \bar{b}))$ be a $K$-variety in $\tau(V)$. Then there exists a tuple $\bar{c}$ in $K(\bar{a}, \bar{b})^{n}$ and a derivation $D^{\star}$ on $K(\bar{a}, \bar{b})$ extending $D_{K}$ such that $D^{\star}(\bar{a}, \bar{b})=(\bar{b}, \bar{c})$.

Proof This lemma directly follows from [6], Theorem 1.1 and Corollary 1.7.

\section{Model Companion of Theories of Differential Topological Fields}

In this section we generalize the construction of Pierce and Pillay for $D C F_{0}$ to obtain a geometric axiomatization for the model companion of some theories of differential topological fields which will be defined in Definition 2.8. From now on we consider a first-order extension $\mathcal{L}$ of the language $\mathscr{L}_{\text {fields }}$ which contains no other function symbol than the ones appearing in $\mathscr{L}_{\text {fields }}$ (i.e., $+, \cdot,-$, , and ${ }^{-1}$ ) and an $\mathcal{L}$-theory $T$ expanding the theory of fields of characteristic zero. The following definition is a special case of the definition of a first-order topological structure introduced by Pillay in [7].

Definition 2.1 Let $K$ be a model of $T$ and $\varphi(X ; \bar{Y})$ be an $\mathcal{L}$-formula.

1. $\langle K, \varphi\rangle$ is a (first-order) topological $(\mathcal{L})$-field if the $\operatorname{set}\left\{\varphi(X ; \bar{k}) ; \bar{k} \in K^{n}\right\}$ of $K$-definable subsets of $K$ is a basis for a topology $\tau_{\varphi}$ on $K$.

2. $\langle T, \varphi\rangle$ is a (first-order $\mathcal{L}$-) theory of topological fields if any model of $T$ is a topological field (equipped with the topology $\tau_{\varphi}$ ).

Note that if $L \equiv K$ then $\langle L, \varphi\rangle$ is also a first-order topological $\mathcal{L}$-field. 


\section{Example 2.2}

1. Let $\langle K, \leq\rangle$ be an ordered field where $\leq$ is a total ordering on $K$. Then $\langle K, \varphi\rangle$ is a topological $\mathscr{L}_{\leq}$-field where $\mathscr{L}_{\leq}:=\mathscr{L}_{\text {fields }} \cup\{\leq\}$ and $\varphi$ is the following formula:

$$
\varphi\left(X ; k_{1}, k_{2}\right):=k_{1}<X \wedge X<k_{2} \quad \text { where } k_{1}, k_{2} \in K .
$$

Note that the topology $\tau_{\varphi}$ is the usual order topology on $K$.

2. Let $\langle K, v\rangle$ be a nontrivially valued field. We consider the valuation topology on $K$ which is defined by the formula

$$
\varphi\left(X ; k_{1}, k_{2}\right):=v\left(k_{1}\right)<v\left(X-k_{2}\right) \text { where } k_{1}, k_{2} \in K .
$$

Hence $\langle K, \varphi\rangle$ is a topological $\mathcal{L}_{\mathscr{D}}$-field where $\mathcal{L}_{\mathscr{D}}:=\mathcal{L}_{\text {fields }} \cup\{\mathscr{D}\}$ and $\mathscr{D}$ is the linear divisibility relation corresponding to the valuation $v$ (i.e., $\forall a, b \in K \mathscr{D}(a, b)$ if and only if $v(a) \leq v(b))$.

Furthermore, we impose some additional conditions on the topology $\tau_{\varphi}$. For this we introduce the two following definitions.

Definition 2.3 Let $\langle K, \varphi\rangle$ be a topological field. Then $\langle K, \varphi\rangle$ is proper if

1. the topology $\tau_{\varphi}$ on $K$ is $T_{1}$ (i.e., for any $k_{1}, k_{2} \in K$ with $k_{1} \neq k_{2}$, there exists a basic open set $U \subseteq K$ such that $k_{1} \in U$ and $k_{2} \notin U$ );

2. $K$ has no isolated points in the topology $\tau_{\varphi}$.

Lemma 2.4 If $\langle K, \varphi\rangle$ is proper then any open subset of $K$ is infinite.

Proof Assume $\left\{a_{1}, \ldots, a_{l}\right\}$ is a finite open subset of $K$. Since $\tau_{\varphi}$ is $T_{1}$ there exists, for each $i \in\{1, \ldots, l-1\}$, an open subset $U_{i}$ of $K$ such that $a_{n} \in U_{i}$ and $a_{i} \notin U_{i}$. Hence $\left(\bigcap_{i=1}^{l-1} U_{i}\right) \cap\left\{a_{1}, \ldots, a_{l}\right\}=\left\{a_{n}\right\}$ is open, contradicting the fact that $K$ has no isolated point with respect to $\tau_{\varphi}$.

Definition 2.5 ([12]) $\quad$ Let $\langle K, \varphi\rangle$ be a proper topological $\mathcal{L}$-field. Then $K$ is a topological $(\mathcal{L}-)$ system if

1. for every $n$-ary function symbol $f$ of $\mathscr{L}_{\text {fields }}(n \in\{1,2\}), f^{K}: K^{n} \longmapsto K$ is a continuous function with respect to $\tau_{\varphi}$ (in particular, each $\mathcal{L}$-term is continuous with respect to $\tau_{\varphi}$ );

2. for every $m_{i}$-ary relation $R_{i}$ of $\mathcal{L}\left(m_{i} \in \mathbb{N}\right)$ and for all sequences $1 \leq i_{1} \leq \cdots \leq i_{l} \leq m_{i}$, the definable sets

$$
\left\{\left(k_{i_{1}}, \ldots, k_{i_{l}}\right) \in K^{l}: R_{i}^{K}\left(\frown, k_{i_{1}}, \frown, k_{i_{l}}, \frown\right) \wedge \bigwedge_{r=1}^{l} k_{i_{r}} \neq 0\right\}
$$

and

$$
\left\{\left(k_{i_{1}}, \ldots, k_{i_{l}}\right) \in K^{l}: \neg R_{i}^{K}\left(\frown, k_{i_{1}}, \frown, k_{i_{l}}, \frown\right) \wedge \bigwedge_{r=1}^{l} k_{i_{r}} \neq 0\right\}
$$

are open in $K^{l}$ (where $\frown$ stands for the tuple $\left.\overline{0}\right)$.

Remark 2.6 Ordered fields and valued fields, considered as topological fields as in Examples 2.2, are topological systems, respectively, in the languages $\mathscr{L}_{\leq}$and $\mathscr{L}_{\mathscr{D}}$ described above (see [4], Chapter 4). 
In what follows we will restrict ourselves to the topological fields which satisfy Definitions 2.3 and 2.5. In other words, we assume that all the topological $\mathcal{L}$-fields $K$ we consider are proper topological $\mathcal{L}$-systems (in the same way an $\mathcal{L}$-theory $T$ is a theory of topological field if all its models are topological $\mathscr{L}$-fields which are proper topological $\mathcal{L}$-systems). This hypothesis has a direct interesting consequence on the structure of quantifier-free $\mathcal{L}$-definable sets in $K$.

Lemma 2.7 Let $K$ be a topological $\mathcal{L}$-field. Then every quantifier-free definable subset $A \subset K^{n}$ is a finite union of subsets of $K^{n}$ which are an intersection of a $K$-variety with a $K$-definable open subset of $K^{n}$.

Proof This is an easy corollary of Definitions 2.3 and 2.5 (see also [4], Chap. 4).

We will now assume that the topological fields we consider are equipped with a derivation.

Definition 2.8 Let $T$ be an $\mathcal{L}$-theory of topological fields; we denote by $T^{D}$ the $\mathscr{L}^{D}$-theory ${ }^{1}$ obtained by adding to $T$ the usual axioms for the derivation (i.e., $\forall X, Y \quad D(X+Y)=D(X)+D(Y) \wedge D(X Y)=D(X) Y+X D(Y)$ ). The models of $T^{D}$ are called differential topological $(\mathcal{L})$-fields.

Our goal now is to build the model companion $\left(T^{D}\right)^{*}$ of the $\mathcal{L}^{D}$-theory $T^{D}$ under the assumption that the $\mathcal{L}$-theory $T$ already has a model companion that we denote by $T^{*}$.

The following definition gives a scheme of axioms which characterizes the existentially closed models of $T^{D}$. Unfortunately we have to ensure the satisfaction of a given condition (Condition 1) which is a priori not first-order in $\mathcal{L}\left(\right.$ or $\mathscr{L}^{D}$ ). Nevertheless, we will see in the next section how to replace Condition 1 in order to obtain first-order axioms for $\left(T^{D}\right)^{*}$ in the special case where the theory $T^{D}$ is large (see Definition 3.4).

In the sequel and similarly to the previous section, if $K$ is a differential topological field then $\Omega$ is a sufficiently saturated $\mathcal{L}$-elementary extension of $K$ (in particular, $\Omega \models T$ ) and $\widetilde{\Omega}$ is the algebraic closure of $\Omega$.

Definition 2.9 Let $\left(K, D_{K}\right) \models T^{D}$; we say that $K \models P P(T)$ if and only if

1. $K \models T^{*}$;

2. for every $K$-variety $V \subseteq \tilde{\Omega}^{n}$ and $W \subseteq \tau(V)$ such that $W$ projects generically $^{2}$ on $V$, if $U$ is a $K$-definable subset of $W$ which satisfies Condition 1 below, then there exists $\bar{c}$ in $\left.V\right|_{K}$ such that $\left.\left(\bar{c}, D_{K}(\bar{c})\right) \in U\right|_{K}{ }^{3}$.

Condition 1 The $K$-definable open subset $U$ of $W$ contains a generic point $(\bar{a}, \bar{b})$ of $W$ in a model $L$ of $T$ extending $K$.

Theorem 2.10 Assume that Condition 1 is expressible in the first-order language $\mathcal{L}$, then $P P(T)$ is the model companion of $T^{D}$ (i.e., $\left.P P(T)=\left(T^{D}\right)^{*}\right)$.

Proof (1) We first show that the models of $P P(T)$ are exactly the existentially closed models of $T^{D}$. This is done in a very similar way as in the case of $D C F_{0}$ (see [6], Theorem 2.1); the main difference is that we have to use Condition 1 to ensure the existence of generic points in the $K$-definable open subsets of $W$ (in the case of $D C F_{0}$ each $K$-definable open subset is $K$-Zariski-open and then contains generic points). 
Let $\left(K, D_{K}\right)$ be an existentially closed model of $T^{D}$ and let us consider an instance of the scheme of axioms $P P(T)$. If Condition 1 holds then there exists a model $M$ of $T$ which extends $K$ and contains a generic point $(\bar{a}, \bar{b})$ of $W$. By Lemma 1.1, we can extend the derivation $D_{K}$ on $K$ to a derivation $D_{M}$ on $M$ such that $D_{M}(\bar{a})=\bar{b}$. Hence,

$$
\left(M, D_{M}\right) \models(\exists \bar{X}, \bar{Y})\left((\bar{X}, \bar{Y}) \in U \wedge D_{M}(\bar{X})=\bar{Y}\right) .
$$

Since $\left(K, D_{K}\right)$ is an existentially closed model of $T^{D}$,

$$
\left(K, D_{K}\right) \models(\exists \bar{X}, \bar{Y})\left((\bar{X}, \bar{Y}) \in U \wedge D_{K}(\bar{X})=\bar{Y}\right),
$$

and the axioms in $P P(T)$ hold in $\left(K, D_{K}\right)$.

Let us assume now that $\left(K, D_{K}\right)$ is a model of $P P(T)$ and consider an extension $\left(M, D_{M}\right)$ of $\left(K, D_{K}\right)$ and a tuple $\bar{a} \subset M$. Let $\varphi(\bar{X})$ be a quantifier free $\mathcal{L}^{D}$-formula with parameters from $K$ such that $\left(M, D_{M}\right) \models \varphi(\bar{a})$. Note that $\varphi(\bar{X})$ is equivalent to

$$
\varphi_{\mathcal{L}}\left(\overline{X_{0}}, \overline{X_{1}}, \ldots, \overline{X_{r}}\right) \wedge \overline{X_{1}}=D\left(\overline{X_{0}}\right) \wedge \cdots \wedge \overline{X_{r}}=D\left(\overline{X_{r-1}}\right),
$$

where $\varphi_{\mathscr{L}}$ is an $\mathcal{L}$-formula. ${ }^{4}$ Define $\Phi_{\mathscr{L}}(\bar{X}, \bar{Y})$ to be the following $\mathcal{L}$-formula:

$$
\Phi_{\mathcal{L}}(\bar{X}, \bar{Y}) \equiv \varphi_{\mathcal{L}}\left(\overline{X_{0}}, \overline{X_{1}}, \ldots, \overline{X_{r-1}}, \overline{Y_{r-1}}\right) \wedge \overline{X_{1}}=\overline{Y_{0}} \wedge \cdots \wedge \overline{X_{r-1}}=\overline{Y_{r-2}} .
$$

Let $\bar{c}=\left(\bar{a}, D_{M}(\bar{a}), \ldots, D_{M}{ }^{(r-1)}(\bar{a})\right), V=V(I(\bar{c}))$, and $W=V\left(I\left(\bar{c}, D_{M} \bar{c}\right)\right)$ (so that $W$ projects generically onto $V$ ). By Lemma 2.7 we may assume that the set defined by $\Phi_{L}$ in $M$ is a finite union of subsets which are the intersection of a $K$-variety and a $K$-definable open set. One of these open subsets, say $U$, contains $\left(\bar{c}, D_{M}(\bar{c})\right)$. Hence, by the scheme of axioms $P P(T)$, there exists a point $\left(\bar{d}, D_{K}(\bar{d})\right)$ in $\left.U\right|_{K}$ so that $K \models \varphi_{\mathscr{L}}\left(\bar{d}, D_{K}(\bar{d})\right)$. Hence $K \models \varphi\left(\bar{d}_{0}\right)$ where $\bar{d}_{0}$ is the initial subtuple of length equal to the length of $\bar{a}$.

(2) We have now to show that any model of $T^{D}$ extends to a model of $P P(T)$. Extending the derivation if necessary, we may assume without loss of generality that $K \models T^{*}$. Let $V, W, U$ be as in Definition 2.9. By Condition 1, $U$ contains a generic point $(\bar{a}, \bar{b})$ of $W$ in a model $M$ of $T$ extending $K$. By Lemma 1.1, the derivation on $K$ extends to a derivation $D_{M}$ on $M$ such that $D_{M}(\bar{a})=\bar{b}$. Since $T$ is an inductive $\mathcal{L}$-theory, one can build a model of $P P(T)$ by using a classical argument of transfinite chains.

The following corollary gives the condition under which this axiomatization provides a way to construct the model completion of a theory of differential topological fields.

Corollary 2.11 If we suppose furthermore that $\left(T^{D}\right)_{\forall}$ has the amalgamation property (and then $T_{\forall}$ also has) then $P P(T)$ has quantifier elimination in $\mathcal{L}^{D}$ and is the model completion of $T^{D}$.

Proof This is a direct consequence of Theorem 2.10 and of the fact that a theory $T$ has quantifier elimination if and only if $T$ is model complete and $T_{\forall}$ has the amalgamation property (see the proof of [3], Theorem 8.4.1).

\section{Large Differential Fields and Condition 1}

As said before the scheme of axioms $P P(T)$ only characterizes the existentially closed models of $T^{D}$ if Condition 1 is satisfied and this condition is not, a priori, 
first-order in $\mathcal{L}^{D}$. Our aim here is to get rid of Condition 1 and to exhibit a scheme of axioms which is first-order in $\mathcal{L}^{D}$.

We first recall a definition introduced in [5]: Let $\bar{a}=\left(a_{1}, \ldots, a_{n}\right) \in \widetilde{\Omega}^{n}$ be a generic point of a $K$-variety $V \subset \widetilde{\Omega}^{n}$. We can suppose that $a_{1}, \ldots, a_{r}$ are algebraically independent over $K$ and that the $a_{r+i}$ s are algebraic over $K\left(a_{1}, \ldots, a_{r}\right)$ for all $i \in\{1, \ldots, n-r\}$. Let $Q_{i}$ be the polynomial obtained from the minimal polynomial of $a_{r+i}$ over $K\left(a_{1}, \ldots, a_{r+i-1}\right)$ after replacing $a_{1}, \ldots, a_{r+i-1}$ by the variables $X_{1}, \ldots, X_{r+i-1}$ and clearing the denominators.

Definition 3.1 These $Q_{i}$ s are called canonical generator ${ }^{5}$ of $V$. Furthermore, we say that a point $\bar{b}$ of $V$ is semi-generic (with respect to the $Q_{i} \mathrm{~s}$ ) if for each $i \in\{1, \ldots, n-r\}$,

$$
s_{Q_{i}}(\bar{b}):=\frac{\partial Q_{i}}{\partial X_{r+i}}(\bar{b}) \neq 0 \wedge H\left(Q_{1}, \ldots, Q_{n-r}\right)(\bar{b}) \neq 0,
$$

where $H\left(Q_{1}, \ldots, Q_{n-r}\right)$ is the product of the dominant coefficient of each $Q_{i}$ seen as a polynomial in $X_{r+i}$ with coefficients in $K\left[X_{1}, \ldots, X_{r+i-1}\right]$.

In [5] the authors use the following lemma to give a less constrained and first-order version of Condition 1 in the particular case of ordered differential fields.

Lemma 3.2 Let $K$ be a real closed field and assume that $V, W, U$ are as in Definition 2.9. If $\left.U\right|_{K}$ contains a semi-generic point of $W$ then $U$ contains a generic point $(\bar{a}, \bar{b})$ of $W$ such that $K(\bar{a}, \bar{b})$ is an ordered field.

Proof See [5].

The result stated in Lemma 3.2 can also be obtained in other examples of theories of topological fields. For this, we have to remark that the main tool used in the proof of Lemma 3.2 in [5] is the intermediate value property for real closed fields. So this proof can be slightly modified in order to apply to any theory of topological fields $T$ satisfying a topological analogue of this property.

To formalize this we introduce the following two definitions.

Definition 3.3 Let $\langle K, \varphi\rangle$ be a topological $\mathcal{L}$-field and $\Omega$ be a sufficiently saturated $\mathcal{L}$-elementary extension of $K$. An element $t$ of $\Omega$ is $K$-infinitesimal (in $\Omega$ ) if it belongs to all nonempty $K$-definable open subsets of $K$ containing 0 .

Definition 3.4 An $\mathcal{L}$-theory of topological fields $T$ is large if, for any $K, \Omega \models T$ such that $\Omega$ is a sufficiently saturated $\mathcal{L}$-elementary extension of $K$, the following holds: for each $f\left(X_{1}, \ldots, X_{n}, X_{n+1}\right) \in K\left[X_{1}, \ldots, X_{n}, X_{n+1}\right]$, if there exist $y_{1}, \ldots, y_{n} \in \Omega$ and $x \in K$ such that $f\left(y_{1}, \ldots, y_{n}, x\right)$ and $\frac{\partial f}{\partial X_{n+1}}\left(y_{1}, \ldots, y_{n}, x\right)-k$ are $K$-infinitesimal (for some $k \in K \backslash\{0\}$ ), then there exists $y \in \Omega$ such that $f\left(y_{1}, \ldots, y_{n}, y\right)=0$ and $x-y$ is $K$-infinitesimal. We will also say that the $\mathcal{L}^{D}$ theory $T^{D}$ is large when $T$ is.

We now generalize Lemma 3.2 to the case of large theories of topological fields.

Lemma 3.5 Let $K \models T$ where $T$ is a large $\mathcal{L}$-theory of topological fields and let $V \subseteq \tilde{\Omega}^{n}$ be a $K$-variety. Assume, furthermore, that $W \subseteq \tau(V)$ is a $K$-variety which projects generically onto $V$. If $U \subset W$ is a nonempty $K$-definable open set such that $\left.U\right|_{K}$ contains a semi-generic point of $W$ then $U$ contains a generic point $(\bar{a}, \bar{b})$ of $W$ such that $K(\bar{a}, \bar{b}) \models T$. 
This lemma simply says that, given a large theory of topological fields $T$ and a model $K$ of $T$, if we assume that $V, W, U$ are as in the scheme of axioms $P P(T)$ then Condition 1 holds as soon as $\left.U\right|_{K}$ contains a semi-generic point for $W$.

Proof As previously noted the proof is a generalization of the proof of Lemma 3.2 appearing in [5] (in this particular case, the topology is the order topology and the role of the largeness hypothesis is played by the intermediate value property for real closed fields).

Note first that since $W$ projects generically onto $W$ we can find a set of canonical generators $\left\{Q_{1}, \ldots, Q_{n-r}, Q_{n+1}, \ldots, Q_{2 n-s}\right\}$ of $W$ where $r=\operatorname{dim} V$, $r+s=\operatorname{dim} W$, and $Q_{1}, \ldots, Q_{n-r}$ are canonical generators of $V$. Let $(\bar{a}, \bar{b})$ be a semi-generic point of $\left.W\right|_{K}$ belonging to $U$ and recall that $\Omega$ is a sufficiently saturated $\mathcal{L}$-elementary extension of $K$.

Claim 3.6 In $\Omega$ we can choose $t_{1}, \ldots, t_{r}, u_{1}, \ldots, u_{s} K$-infinitesimal and algebraically independent over $K$.

Assume the claim is proved and put $c_{l}=a_{l}+t_{l}$ and $d_{m}=b_{m}+u_{m}$ for each $l \in\{1, \ldots, r\}$ and each $m \in\{1, \ldots, s\}$. Since $Q_{1}$ and $Q_{n+1}$ vanish at $(\bar{a}, \bar{b})$, the elements $Q_{1}\left(c_{1}, \ldots, c_{r}, a_{r+1}\right)$ and $Q_{n+1}\left(c_{1}, \ldots, c_{r}, a_{r+1}, \ldots, a_{n}, d_{1}, \ldots, d_{s}, b_{s+1}\right)$ of $\Omega$ are $K$-infinitesimal. Using the largeness of $T$ recursively first on each $a_{r+i}$ and then on each $b_{s+j}$ (with $i \in\{1, \ldots, n-r\}$ and $j \in\{1, \ldots, n-s\}$ ), we find for each $i$ (respectively, each $j$ ) an element $k_{i}$ (respectively, $\tilde{k}_{j}$ ) in $\Omega$ such that $Q_{i}\left(c_{1}, \ldots, c_{r+i-1}, k_{i}\right)=0$ (respectively, $Q_{n+j}\left(\bar{c}, d_{1}, \ldots, d_{s+j-1}, \tilde{k}_{j}\right)=0$ ) and $k_{i}-a_{i}$ (respectively, $\tilde{k}_{j}-b_{j}$ ) are $K$-infinitesimal. Put $c_{r+i}=k_{i}$ and $d_{s+j}=\tilde{k}_{j}$ for all $i \in\{1, \ldots, n-r\}$ and $j \in\{1, \ldots, n-s\}$; then $\left.(\bar{c}, \bar{d}) \in W\right|_{K}$ is $K$-infinitesimally close to $(\bar{a}, \bar{b})$ (i.e., the tuple $(\bar{c}-\bar{a}, \bar{d}-\bar{b})$ is $K$-infinitesimal). Moreover, the algebraic independence of $t_{1}, \ldots, t_{r}, u_{1}, \ldots, u_{s}$ ensures that $(\bar{c}, \bar{d})$ is a generic point of $W$.

Proof of the claim Let us consider the following set $F\left(X_{1}, \ldots, X_{r+s}\right)$ of all formulas:

$$
f\left(X_{1}, \ldots, X_{r+s}\right) \neq 0 \wedge \bigwedge_{i=1}^{r+s} \varphi\left(X_{i} ; \bar{k}\right),
$$

where $f\left(X_{1}, \ldots, X_{r+s}\right)$ ranges over nonzero polynomials in $r+s$ indeterminates with coefficients in $K, \varphi$ is the distinguished $\mathcal{L}$-formula which determines a basis of the topology, and the tuple $\bar{k}$ ranges over $K$ and is such that $\varphi(K ; \bar{k}):=\{x \in K \mid K \models \varphi(x, \bar{k})\}$ contains 0 (let us denote by $K_{0}$ the set of such tuples). By the saturation of $\Omega$, it is sufficient to show that this set is a partial $(r+s)$ type over $K$. In other words, it is sufficient to prove that there exist $x_{1}, \ldots, x_{r+s}$ in $\Omega$ such that

$$
f\left(x_{1}, \ldots, x_{r+s}\right) \neq 0 \wedge \bigwedge_{i=1}^{r+s} \varphi\left(x_{i} ; \bar{k}_{0}\right) \text { for a given tuple } \bar{k}_{0} \in K_{0} .
$$

We proceed by induction on $r+s$. If $r+s=1$ the result follows since $f(X)$ has only finitely many roots in $K$ and, by Lemma $2.4, \varphi\left(K ; \bar{k}_{0}\right)$ is infinite. Suppose now that there exist $x_{1}, \ldots, x_{r+s-1}$ in $\Omega$ such that for all $\vec{k}$ in $K_{0}$ and for each nonzero 
polynomial $f\left(X_{1}, \ldots, X_{r+s-1}\right)$ with coefficients in $K$ we get

$$
f\left(x_{1}, \ldots, x_{r+s-1}\right) \neq 0 \wedge \bigwedge_{i=1}^{r+s-1} \varphi\left(x_{i} ; \bar{k}\right) .
$$

Let $g\left(X_{1}, \ldots, X_{r+s}\right)$ be a nonzero polynomial in $r+s$ indeterminates with coefficients in $K$ and fix a tuple $\bar{k}_{0}$ in $K_{0}$. If

$$
\forall x_{1}, \ldots, x_{r+s} \in \varphi\left(\Omega, \bar{k}_{0}\right) \quad g\left(x_{1}, \ldots, x_{r+s}\right)=0
$$

then, by the inductive hypothesis, $g\left(x_{1}, \ldots, x_{r+s-1}, X\right) \equiv 0$ for $x_{1}, \ldots, x_{r+s-1}$ belonging to $\varphi\left(K ; \bar{k}_{0}\right)$. Therefore, since $K \prec \Omega$,

$$
\Omega \models \forall x_{1}, \ldots, x_{r+s-1}\left(\bigwedge_{i=1}^{r+s-1} \varphi\left(x_{i} ; \bar{k}_{0}\right) \Rightarrow g_{i}\left(x_{1}, \ldots, x_{r+s-1}\right)=0\right)
$$

where $g=\sum_{i=1}^{d} g_{i} \cdot X_{n}^{i}$ for some natural number $d$. Using again the inductive hypothesis one can deduce that $g_{i} \equiv 0$ and hence $g \equiv 0$, a contradiction.

This last lemma allows us to restate, in this special case of large theories, the axioms in 2.9 in the following first-order way.

Theorem 3.7 Let $T$ be a large $\mathcal{L}$-theory of topological fields which admits a model companion $T^{*}$ and let $K \models T^{D}$. Then $\left(K, D_{K}\right) \models\left(T^{D}\right)^{*}$ if and only if

1. $K \models T^{*}$;

2. for all $K$-varieties $V$ and $W \subset \tau(V)$ such that $W$ projects generically onto $V$, if $U \subset W$ is a nonempty $K$-definable open subset such that $\left.U\right|_{K}$ contains a semi-generic point of $W$ then $U$ contains a point of the form $\left(\bar{a}, D_{K}(\bar{a})\right)$.

\section{Example 3.8}

1. The largeness of the $\mathscr{L}_{\leq}$-theory $R C F$ (seen as a theory of topological fields as in Examples 2.2) follows easily from the intermediate value property. Hence Theorem 3.7 applies to ordered differential fields and allows to recover the geometric axiomatization of the theory of closed ordered differentially fields $(C O D F)$ obtained in [5].

2. On the other hand, the work developed in [2] shows that henselian nontrivially valued fields are also large in the sense of Definition 3.4. Hence Theorem 3.7 provides a geometric analogue to the axiomatization of the theory $\left(A C V F_{0}\right)_{D}^{*}$ introduced in [2], Theorem 4.2 and Corollary 5.2.

We end this section with a few words about the first-order expressibility of the axiomatization appearing in Theorem 3.7 in the language $\mathcal{L}^{D}$. Let $I(V)=$ $\left\langle f_{1}(\bar{X}), \ldots, f_{m}(\bar{X})\right\rangle$ and $I(W)=\left\langle g_{1}(\bar{X}, \bar{Y}), \ldots, g_{s}(\bar{X}, \bar{Y})\right\rangle$. Choose a tuple $\bar{u}$ and polynomials $F_{i}(\bar{U}, \bar{X}) \in \mathbb{Z}[\bar{U}, \bar{X}], G_{j}(\bar{U}, \bar{X}, \bar{Y}) \in \mathbb{Z}[\bar{U}, \bar{X}, \bar{Y}]$ such that $f_{i}(\bar{X})=F_{i}(\bar{u}, \bar{X})$ for $i=1, \ldots, m$ and $g_{j}(\bar{X}, \bar{Y})=G_{j}(\bar{u}, \bar{X}, \bar{Y})$ for $j=1, \ldots, s$. By the work of van den Dries and Schmidt in [11], the following properties of the tuple $\bar{u}$ are expressible by a first-order formula:

1. $F_{1}(\bar{u}, \bar{X}), \ldots, F_{m}(\bar{u}, \bar{X})$ generate a prime ideal $I(V)$ in $K[\bar{X}]$;

2. $G_{1}(\bar{u}, \bar{X}, \bar{Y}), \ldots, G_{s}(\bar{u}, \bar{X}, \bar{Y})$ generate a prime ideal $I(W)$ in $K[\bar{X}, \bar{Y}]$ which intersects $K[\bar{X}]$ in $I(V)$. 
An other important point is to express (by a first-order formula in the variables $\bar{u}$ ) that given a set of polynomials $Q_{1}(\bar{U}, \bar{X}, \bar{Y}), \ldots, Q_{t}(\bar{U}, \bar{X}, \bar{Y}), H(\bar{U}, \bar{X}, \bar{Y}) \in \mathbb{Z}[\bar{U}, \bar{X}]$ (in our case the canonical generators of the ideal $I(W))$, the ideal $\left\langle Q_{1}(\bar{u}, \bar{X}, \bar{Y})\right.$, $\left.\ldots, Q_{t}(\bar{u}, \bar{X}, \bar{Y})\right\rangle: H(\bar{u}, \bar{X}, \bar{Y})^{\infty}$ is prime and is equal to $I(W)$. This also can be deduced from [11] and the following fact (see [10]).

Fact 3.9 Let $I$ be an ideal of a domain $A$ and $h \in A$; then

$$
I: h^{\infty}=\langle I, Z \cdot h-1\rangle_{A[Z]} \cap A \text { (where } Z \text { is a new indeterminate). }
$$

The reader can refer to [1] to get more details.

Remark 3.10 Finally let us mention that at least two other notions of large theories are related to ours. The first one was introduced by Pop [8] in his solution to the conjecture that asserts that the absolute Galois group of a countable, Hilbertian, PAC field is profinite free. It was recently used by Tressl to give a uniform axiomatization of model companion of theories of differential fields in several derivations [10]. The second notion of large is introduced by the first author and Point in [2], Definition 2.4, in their study of model companions of topological theories of differential fields. Let us remark that our method to uniformly build model companions for theories of differential fields is quite different from the ones used in [10] and [2] since it is more related to the Pierce-Pillay method. One of the advantages of this method is that one does not need differential algebra to axiomatize theories of differential fields but only classical objects from algebraic geometry.

\section{Existentially Closed Differential Fields and Jet-Spaces}

In this last section we use the material introduced before to prove that in the existentially closed models of a large $\mathcal{L}^{D}$-theory $T^{D}$ of differential topological fields, the jet-spaces are dense in their ambient space with respect to the underlying topology $\tau_{\varphi}$ of $T$.

We first recall the definition of the jet-space associated with a differential field.

Definition 4.1 Let $K$ be a differential field and $\left(r_{1}, \ldots, r_{n}\right)$ be a tuple of positive integers; the $\left(r_{1}, \ldots, r_{n}\right)$-jet-space of $K$ is the set

$$
J_{\left(r_{1}, \ldots, r_{n}\right)}(K)=\left\{\left(x_{1}, x_{1}^{\prime}, \ldots, x_{1}{ }^{\left(r_{1}\right)}, \ldots, x_{n}, x_{n}^{\prime}, \ldots, x_{n}{ }^{\left(r_{n}\right)}\right) \mid\left(x_{1}, \ldots, x_{n}\right) \in K^{n}\right\} .
$$

Theorem 4.2 Let $\left\langle K, \varphi, D_{K}\right\rangle \models T^{D}$ where $T^{D}$ is a large $\mathcal{L}^{D}$-theory of differential topological fields. If $K$ is an existentially closed model of $T^{D}$ (i.e., $K$ is a model of $P P(T))$ then for each positive integer $n$ and each $\left(r_{1}, \ldots, r_{n}\right) \in \mathbb{N}^{n}, J_{\left(r_{1}, \ldots, r_{n}\right)}(K)$ is dense in $K^{\left(r_{1}+1\right)+\cdots+\left(r_{n}+1\right)}$ with respect to the product topology induced by $\tau_{\varphi}$ on $K^{\left(r_{1}+1\right)+\cdots+\left(r_{n}+1\right)}$.

Proof Since $J_{\left(r_{1}, \ldots, r_{n}\right)}(K)=J_{r_{1}}(K) \times \cdots \times J_{r_{n}}(K)$ and density is preserved by direct products of topological spaces it is sufficient to prove that, for any positive integer $n>1$, the $(n-1)$-jet-space of $K$ is dense in $K^{n}$. For this let $U$ be a $K$ definable open subset of $K^{n}$ in order to show that $U$ contains a point of $J_{n-1}(K)$. Consider the differential polynomial $f(X)=X^{(n)}$ and the following varieties:

$$
\left\{\begin{array}{l}
V:=V((0)) \\
W:=\left\{(\bar{X}, \bar{Y}) \mid f\left(X_{0}, \ldots, X_{n-1}, Y_{n-1}\right)=0 \wedge Y_{0}=X_{1} \wedge \cdots \wedge Y_{n-2}=X_{n-1}\right\} .
\end{array}\right.
$$


Let us note that $W$ projects generically over $V$. Let $\widetilde{U}$ be the $K$-definable open subset $\left\{\left(u_{o}, \ldots, u_{n-1}, u_{1}, \ldots, u_{n-1}, 0\right) \mid\left(u_{o}, \ldots, u_{n-1}\right) \in U\right\}$ of $W$. It is easy to see that each point of $\widetilde{U}$ is semi-generic for $W$ and then, using the fact that $T$ is large (Lemma 3.5 applies) and axiom 2 of Theorem 3.7, we get a differential point $\left(u, u^{\prime}, \ldots, u^{(n-1)}\right)$ in $U$ proving the density of the jet-space.

\section{Notes}

1. The language $\mathcal{L}^{D}$ is equal to $\mathcal{L} \cup\{D\}$ where $D$ is a function symbol which is interpreted as the derivation in the models of $T^{D}$.

2. This means that for any generic point $\bar{c}$ of $V$ there exists $\bar{d} \in \tilde{\Omega}^{n}$ such that $(\bar{c}, \bar{d})$ is a generic point of $W$.

3. The set $\left.V\right|_{K}$ (respectively, $\left.U\right|_{K}$ ) is the trace on $K$ of the $K$-variety $V$ (respectively, $K$-definable open set $U$ ).

4. In this context, this assertion means that the $n$-tuple $\bar{X}$ satisfy the $\mathcal{L}^{D}$-formula $\varphi$ if and only if the $(r(n+1))$-tuple $\left(\bar{X}, D(\bar{X}), \ldots, D^{(r)}(\bar{X})\right)$ satisfy the $\mathcal{L}$-formula $\varphi_{\mathcal{L}}$.

5. We use the name "canonical generator" because the ideal defining $V$ is equal to $\left\langle Q_{1}, \ldots, Q_{n-r}\right\rangle: H\left(Q_{1}, \ldots, Q_{n-r}\right)^{\infty}=\left\{f \in K\left[X_{1}, \ldots, X_{n}\right] \mid H\left(Q_{1}, \ldots, Q_{n-r}\right)^{n} \cdot f\right.$ $\in\left\langle Q_{1}, \ldots, Q_{n-r}\right\rangle$ for some natural number $\left.n\right\}$.

\section{References}

[1] Chatzidakis, Z., "Generic automorphisms of separably closed fields," Illinois Journal of Mathematics, vol. 45 (2001), pp. 693-733. Zbl 0993.03047. MR 1879231. 339

[2] Guzy, N., and F. Point, "Topological differential structures," (2004). submitted. 338, 339

[3] Hodges, W., Model Theory, vol. 42 of Encyclopedia of Mathematics and its Applications, Cambridge University Press, Cambridge, 1993. Zbl 0789.03031. MR 1221741. 335

[4] Mathews, L., "Cell decomposition and dimension functions in first-order topological structures," Proceedings of the London Mathematical Society. Third Series, vol. 70 (1995), pp. 1-32. Zbl 0829.03019. MR 1300839. 333, 334

[5] Michaux, C., and C. Rivière, "Quelques remarques concernant la théorie des corps ordonnés différentiellement clos," Bulletin of the Belgian Mathematical Society. Simon Stevin, vol. 12 (2005), pp. 341-48. Zbl 05051871. MR 2173697. 336, 337, 338

[6] Pierce, D., and A. Pillay, "A note on the axioms for differentially closed fields of characteristic zero," Journal of Algebra, vol. 204 (1998), pp. 108-15. Zbl 0922.12006. MR 1623945. 332, 334

[7] Pillay, A., "First order topological structures and theories," The Journal of Symbolic Logic, vol. 52 (1987), pp. 763-78. Zbl 0628.03022. MR 902989. 332 
[8] Pop, F., "Embedding problems over large fields," Annals of Mathematics. Second Series, vol. 144 (1996), pp. 1-34. Zbl 0862.12003. MR 1405941. 339

[9] Shafarevich, I. R., Basic Algebraic Geometry. 2 vols., 2d edition, Springer-Verlag, Berlin, 1994. Zbl 0797.14001. Zbl 0797.14002. MR 1328833. MR 1328834. 331

[10] Tressl, M., "The uniform companion for large differential fields of characteristic 0," Transactions of the American Mathematical Society, vol. 357 (2005), pp. 3933-51 (electronic). Zbl 1077.03019. MR 2159694. 339

[11] van den Dries, L., and K. Schmidt, "Bounds in the theory of polynomial rings over fields. A nonstandard approach,” Inventiones Mathematicae, vol. 76 (1984), pp. 77-91. Zbl 0539.13011. MR 739626. 338, 339

[12] van den Dries, L., "Dimension of definable sets, algebraic boundedness and Henselian fields. Stability in Model Theory, II (Trento, 1987)," Annals of Pure and Applied Logic, vol. 45 (1989), pp. 189-209. Zbl 0704.03017. MR 1044124. 333

\title{
Acknowledgments
}

The authors are supported by an FNRS grant. We would like to thank the referee and Anand Pillay for their wise remarks on this work and for their valuable advice for improving the presentation of this paper. We also thank Christian Michaux for his help during the writing of this paper.

\author{
Université de Mons-Hainaut \\ Institut de Mathématique \\ 6, Avenue du Champ de Mars \\ 7000 Mons \\ BELGIUM \\ nicolas.guzy@umh.ac.be \\ cedric.riviere@umh.ac.be
}

